\title{
Short Tethers for Electrodynamic Thrust
}

\author{
R. D. Estes', E. C. Lorenzini', and J. R. Sanmartin ${ }^{2}$

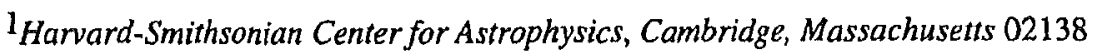 \\ ${ }^{2}$ Escuela Tecnica Superior de Ingenieros Aeronauticos, Universidad Politecnica de Madrid, Spain
}

\begin{abstract}
The operational advantages of electrodynamic tethers of moderate length are becoming evident from studies of collision avoidance. Although long tethers (of order of 10 kilometers) provide high efficiency and good adaptability to varying plasma conditions, boosting tethers of moderate length $(-1$ kilometer) and suitable design might still operate at acceptable efficiencies and adequate adaptability to a changing envitonment. In this paper we carry out a parametric analysis of the performance of $1-\mathrm{km}$ long boosting tethers, to maximize their efficiency. We also discuss the possible use of multiple, parallel such tethers for keeping thrust high when length is decreased. We then estimate the survivability of short tethers to micrometeoroids and orbital debris. Finally, a few considerations are made on the dynamic stability of electrodynamic tether systems versus length.
\end{abstract}

\section{INTRODUCTION}

The simplest figure of merit in comparing space trusters is the ratio of mass $M_{d}$ dedicated to producing thrust, to mission impulse, which is the product of thrust duration $\tau$, and thrust $F$ itself, assumed constant. This ratio should be as small as possible and is just the inverse of specific velocity $v_{s p}$ in the case of chernical propulsion (if tankage and plumbing are neglected). For electrical thrusters, and in particular for lon thrusters, which we shall consider for detailed comparisons, the ratio $M_{d / F} \tau$ involves two terms (Sanmartin, Estes and Lorenzini, 2001),

$$
\begin{gathered}
M_{d} /\left.F \tau\right|_{I}=a_{I}+b_{I} / \tau \\
a_{I} \equiv \frac{1+\alpha}{v_{s p_{I}}}, \quad b_{I} \equiv \beta \times \frac{v_{s p_{I}} / 2}{\eta_{I}} .
\end{gathered}
$$

The first term in (1) is the contribution of propellant mass, as in chemical propulsion, the fraction $\alpha \sim 0.2$ coming from tankage and plumbing. The second term arises from hardware related to the electrical power $W_{e}$. We shall here assume that dedicated solar panels are required in addition to batteries and a power processing unit, resulting in values of $\beta$ of order of $50 \mathrm{~kg} / \mathrm{kw}$. The propulsive efficiency $\eta_{1}$ is typically about 0.5 . For an lon Thruster we also have $v_{S P_{1}} \sim 28 \mathrm{~km} / \mathrm{s}$ (Martinez-Sanchez and Pollard, 1998), which is much greater than chemical specific velocities: ion thrusters attain a much lower ratio $M_{d} / F \tau$ if weak thrusting and long times $\tau \sim b_{l} / a$, are allowed.

But with a few changes, Eqs. (1), (2a, b) apply to a tether too. Now the $b$ term involves the tether mass $M_{t}$ as well as the mass of the power system,

$$
b_{t}=\beta \times \frac{U_{\text {sat }}}{\eta_{t}}, \quad \frac{1}{\eta_{t}}=\frac{W_{e}}{W_{m}}+\frac{\alpha_{t} M_{t}}{\beta W_{m}},
$$

where $W_{m} / W_{e}$ is the propulsive efficiency with $W_{m}=F U_{s a t}$ the mechanical (thrusting) power, and $\eta_{t}$ is an overall tether efficiency that brings into account a measure of thrusting power per unit tether mass (last term in Eq. 3b). The factor $\alpha_{t}-2$ arises from tether-related hardware mass (deployer/end ballast). Note that the role of specific velocity is here played by twice the satellite velocity $U_{\text {sat }}$. 
The $a_{t}$ term arises from expellant mass in a hollow-cathode plasma contactor that ejects electrons at the cathodic end of the tether. The role of specific velocity is now played by the product of a "gyrofrequency"

$$
\omega_{h c} \equiv \frac{I_{h c}}{\dot{m}_{h c}} \times \frac{E_{m}}{U_{s a t}}
$$

and certain effective thrusting length, $W_{m} / E_{m} I_{h c}$ (Sanmartin, Estes and Lorenzini, 2001). In Eq.(4) $E_{m} / U_{s a t}$ is the geomagnetic-field component perpendicular to the orbital plane, $E_{m} \sim 10^{2} \mathrm{~V} / \mathrm{km}$ is the motionally induced electric field, and $I_{h c}$ and $\dot{m}_{h c}$ are current and expellant mass flow rate at the plasma contactor. For state-of-art contactors the ratio $I_{h c} / \dot{m}_{h c}$ has values around the charge-to--mass ratio of singly ionized atoms of atomic number $-4-6$; in LEO orbits at not too high inclination we then have $\omega_{h c}-350 \mathrm{~s}^{-1}$. For effective thrusting lengths (tether length) of order of $10 \mathrm{~km}$, the "specific velocity" in the $a_{t}$ term is 100 times larger than the specific velocity of Ion thrusters: such tethers, over long times, are much more efficient than lon thrusters.

Collision avoidance has recently become a driver for tether design, making the case for shorter tethers. We then note that tethers of length $L_{t}-1 \mathrm{~km}$ would still have a specific impulse 10 times as large as Ion thrusters; they would be more efficient over long enough times. The condition $a_{t}+b_{t} / \tau<a_{1}+b_{t} / \tau$ with $a_{t} \ll a_{t}$ requires

$$
\tau>\frac{b_{I}}{a_{I}}\left(\frac{2 U_{s a t}}{v_{s p I}} \frac{\eta_{I}}{\eta_{t}}-1\right) .
$$

To analyze and design these short tethers and determine operating times one just seeds to maximize $\eta_{t}$. Note that the hollow-cathode bias $\Delta V_{h c}$ might be about $20 \%$ of full induced bias $E_{m} L_{t}$ for $L_{t} \sim 1 \mathrm{~km}$; it cannot be neglected here, as opposed to the 10-km case (Sanmartin, Estes and Lorenzini, 2001).

\section{SHORT BARE-TETHERS FOR THRUSTING}

Figure 1 shows the configuration of a thruster system using a bare tether. At the top there is a power supply to push current down against the motional field $E_{m}$, and electrons off the hollow cathode against its bias. Tether bias $\Delta V$ varies along its length due to both motional and ohmic voltage drop-rates,

$$
\frac{d \Delta V}{d y}=E_{m}+\frac{I}{\sigma_{c} A},
$$

where $\sigma_{C}$ and $A_{t}$ are tether conductivity and cross section area. An upper segment BC of length $L_{i}$ may need to be insulated to maximize the operating efficiency. The bias $\Delta V_{A}$ at the end point $A$ will vary along the orbit. When negative (Fig. 1a), electrons are collected over a segment DB, with $D$ the varying zero-bias point; since current vanishes at $\mathrm{A}$, and (ion) collection in the negatively-biased segment $\mathrm{AD}$ is negligible, we may set $I_{D}=0$. When $\Delta V_{A}$ is positive (Fig. 1b) electrons are collected over the entire segment $A B$.

Along either electron collecting segment, current $I$ will vary according to the OML current-law rate, decreased by a factor $G<1$ if the cross section is too large, or non-convex (Sanmartin and Estes, 2001),

$$
\frac{d l}{d y}=G \times \frac{p}{\pi} e N_{\infty} \sqrt{\frac{2 e \Delta V}{m_{e}}},
$$

with $N_{\infty}$ the ionospheric plasma density and $p$ the perimeter of the tether cross section; the factor $G$ may be taken independent of $y$ because it is nearly independent of bias for values of interest (Sanmartin and Estes, 1999; Estes 
and Sanmartin, 2000). To determine bias and current profiles along the tether one introduces convenient dimensionless variables,

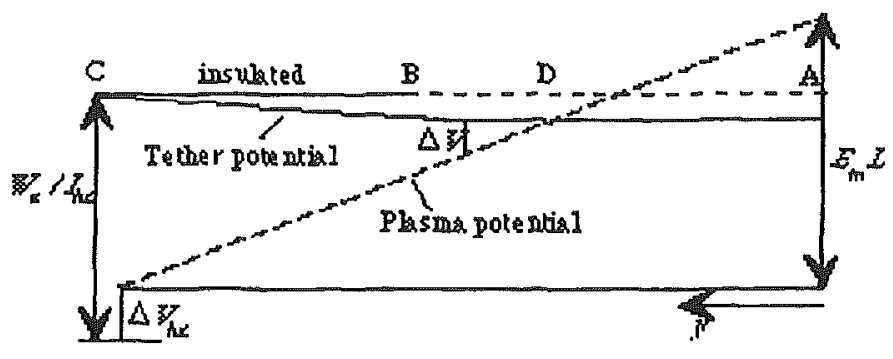

(a)

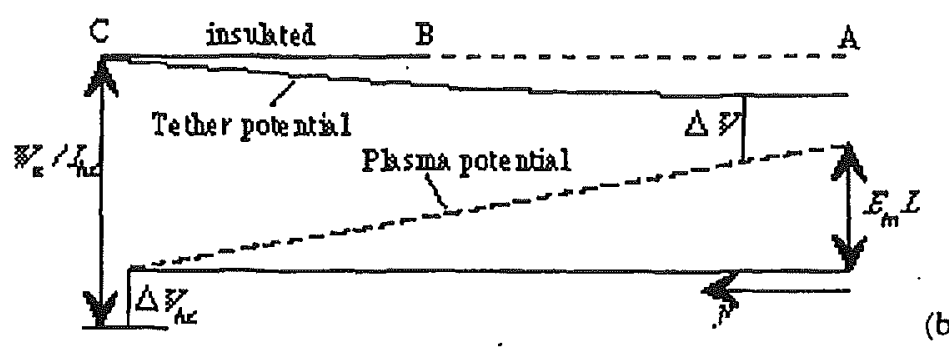

(b)

FIGURE 1. Tether voltage diagrams: (a) $\Delta \mathrm{V}_{\mathrm{A}}<0$; (b) $\Delta \mathrm{V}_{\mathrm{A}}>0$

$$
i \equiv I / \sigma_{c} E_{m} A_{t}, \quad \phi \equiv \Delta V / E_{m} L^{*},
$$

and $y / L^{*}$, with $L^{*}$ (a characteristic length gauging ohmic effects on bare tether collection) defined by

$$
L^{*} \times G \times \frac{p}{\pi} e N_{\infty} \sqrt{\frac{2 e E_{m} L^{*}}{m_{e}}}=\frac{3}{4} \sigma_{c} E_{m} A_{c} \Rightarrow L^{*} \propto\left(\frac{\sigma_{c} \sqrt{E_{m}}}{G N_{\infty}} \frac{A_{t}}{p}\right)^{2 / 3} .
$$

Taking nominal environment conditions $N_{\infty}=3 \times 10^{11} \mathrm{~m}^{-3}, E_{m}=120 \mathrm{~V} / \mathrm{km}$, and a thin tape, aluminum tether in the OML regime $\left(2 A_{t} / p=\right.$ tape thickness $\left.=0.1 \mathrm{~mm}, \sigma_{c}=3.5 \times 10^{7} / \Omega \mathrm{m}, G=1\right)$, we have $L^{*}=1.35 \mathrm{~km}$. The overall efficiency $\eta_{t}$ comes out to be a function of five dimensionless numbers, depending on environmental and tether-system parameters,

$$
\begin{aligned}
\Lambda_{i} \equiv \frac{L_{i}}{L_{t}}, \quad \lambda_{t} \equiv \frac{L^{*}}{L_{t}}, & \tilde{W}_{e} \equiv \frac{W_{e}}{\sigma_{c} E_{m}{ }^{2} A_{t} L_{t}}, \\
\gamma \equiv \frac{\alpha_{t} \rho}{\beta \sigma_{c} E_{m}^{2}}, & \psi_{h c} \equiv \frac{\Delta V_{h c}}{E_{m} L_{t}}
\end{aligned}
$$

where $\rho$ is the tether density.

Analyses by Sanmartin, Estes and Lorenzini (2001) and Sanmartin, Martinez-Sanchez and Ahedo (1993) that ignored $\Delta V_{h c}$ dealt with the cases $\Delta V_{A} \leq 0$ and $\Delta V_{A} \geq 0$, respectively. Results from these analyses can be arranged into a common expression for $\eta_{t}$, while retaining $\Delta V_{h c}$, 


$$
\eta_{t}=\frac{\Lambda_{i} i_{h c}+\lambda_{t} Q}{\gamma+\tilde{W}_{e}}
$$

For $\Delta V_{A} \geq 0$, the auxiliary relations

$$
\begin{gathered}
i_{h c}\left[\Lambda_{i}\left(1+i_{h c}\right)+\lambda_{t} \phi_{B}+\psi_{h c}\right]=\tilde{W}_{e} \\
S\left(\phi_{A}, \phi_{B}\right) \equiv \int_{\phi A}^{\phi_{B}} \frac{d \phi}{\sqrt{1+\phi^{3 / 2}-\phi_{A}^{3 / 2}}}=\frac{1-\Lambda_{i}}{\lambda_{t}}
\end{gathered}
$$

with

$$
i_{h c}\left(\phi_{A}, \phi_{B}\right) \equiv \sqrt{1+\phi_{B}^{3 / 2}-\phi_{A}^{3 / 2}}-1
$$

determine $\phi_{A}, \phi_{B}$ (here, $i_{h c} \equiv I_{h d} / \sigma_{c} E_{m} A_{l}, \phi_{A, B} \equiv \Delta V_{A, B} / E_{m} L^{*}$ ). Use of equations (14) and

$$
Q\left(\phi_{A}, \phi_{B}\right) \equiv \phi_{B}-\phi_{A}-S\left(\phi_{A}, \phi_{B}\right)
$$

in (11) then yields $\eta_{t}\left(\gamma, \psi h c, \lambda_{t}, \Lambda_{i}, \tilde{W}_{e}\right)$. For $\Delta V_{A} \leq 0$, one sets

$$
i_{h c} \rightarrow i_{h c 0}\left(\phi_{B}\right) \equiv i_{h c}\left(0, \phi_{B}\right), \quad Q \rightarrow Q_{0}\left(\phi_{B}\right) \equiv Q\left(0, \phi_{B}\right)
$$

in Eqs.(11, 12). Equation (12) then determines $\phi_{B}$, Eq.(11) again yielding $\eta_{t}\left(\gamma, \psi_{h c}, \lambda_{t}, \Lambda_{i}, \tilde{W}_{e}\right)$. The regime $\Delta V_{A} \leq 0$ requires condition

$$
S_{0}\left(\phi_{B}\right) \equiv S\left(0, \phi_{B}\right) \leq\left(1-\Lambda_{i}\right) / \lambda_{t}
$$

\section{TETHER DESIGN}

Dimensionless ratios $\gamma$ and $\psi_{h c}$ are not free-to-choose; for the tether-system parameters and nominal (environment) parameters above, they would take typical values, $\gamma \sim \psi h c-0.2$. As regards $\lambda_{t}$, with $L^{*}=1.35 \mathrm{~km}$ and the $1-\mathrm{km}$ tether-length range of interest here, a characteristic value would just be $\lambda_{t}-1$. Efficiency decreases with growing $\lambda_{t}$ (Sanmartin, Estes and Lorenzini, 2001); we chose a tape because it has lower ratio $A_{t} / p$ (and thus lower $\lambda_{t}$ at given length $L_{t}$ ) than a round wire of equal perimeter $p$.

Figure 2 shows the overall efficiency $\eta_{t}$ versus $\tilde{w}_{e}$ for $\gamma=\psi h c=0.2, \lambda_{t}=1$, and $\Lambda_{i}=0.2,0.3$, and 0.5 . All curves exhibit a maximum, which occurs near the transition (marked by a dot in the curves) between the $\Delta V_{A}<0$ and $\Delta V_{A}>0$ regimes, lying to left and right, respectively. Low (high) $\tilde{W}_{e}$ values in Eq. (11) correspond to a dominant tether mass (power-system mass) case. The maximum reflects the fact that tether mass $\left(\propto A_{t}\right)$ and ohmic impedance $\left(\propto 1 / A_{t}\right)$ vary with cross section area $A_{t}$ in opposite ways and reduce the overall efficiency at too low or too high values of $\tilde{W}_{e} \propto 1 / A_{t}$.

Note that curves are very flat around the maximum. In addition, the maximum is only weakly dependent on $\Lambda_{i}$. Efficiency considerations, therefore, allow considerable freedom in tether design. Note, finally, that characteristic values $\eta_{t}>0.25$ over a substantial $\Lambda_{i}-\tilde{W}_{e}$ domain in Fig. 2 will result in values $\left(2 U_{s a t} / v_{s p l}\right) \times \eta_{1} / \eta_{t}$ close to unity in Eq. (5); with $b_{l} / a_{l} \sim 375$ days, the 1-km long tether would become more efficient than lon thrusters for thrust times longer than about 1 month. This result, however, is very sensitive to the actual value of overall 
efficiency. A reduced orbital $\eta_{t}$-average, arising from off-nominal conditions found in orbit (Sanmartin, Estes and Lorenzini, 2001), might increase the minimum thrust time to 3-4 months.

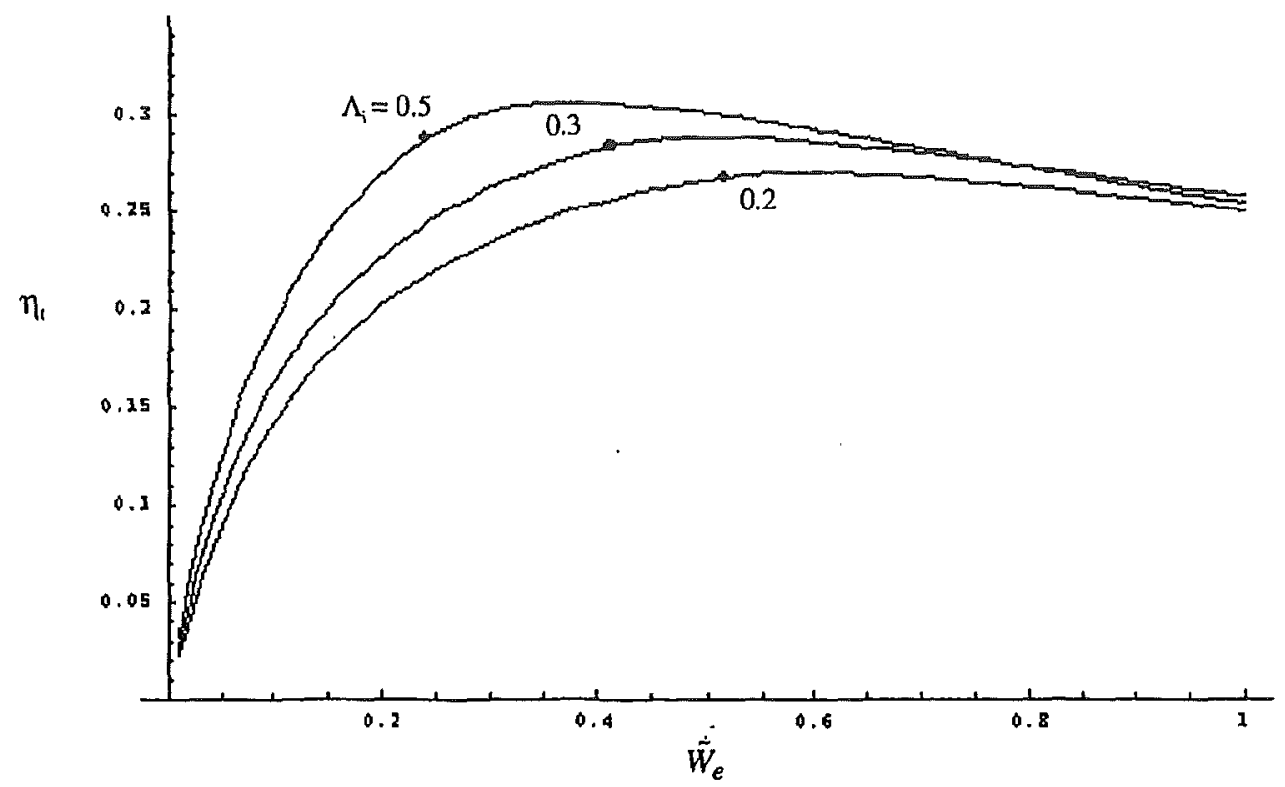

FIGURE 2. Overall tether efficiency vs. dimensionless power $\tilde{W}_{e}$ and insulated length fraction $\Lambda_{1}$

The thrust $F$ now depends on just the cross section $A_{t}$. Using Eqs. (3b) and (11) to write

$$
\frac{1}{\eta_{t}}=\frac{W_{e}}{W_{m}} \times\left[1+\frac{\alpha_{t} M_{t}}{\beta W_{e}}\right] \equiv \frac{\tilde{W}_{e}}{\Lambda_{i} i_{h c}+\lambda_{t} Q} \times\left[1+\frac{\gamma}{\tilde{W}_{e}}\right]
$$

one then finds

$$
\frac{U_{s a t} F}{\sigma_{c} E_{m}{ }^{2} L_{t} A_{t}}=\Lambda_{i} i_{h c}+\lambda_{t} Q=\left(\gamma+\tilde{W}_{e}\right) \eta_{t}
$$

The maximum tape width to ensure OML electron collection is roughly 4 times the Debye length (Sanmartin and Estes, 1999). At the nominal density $\left(N_{\infty}=3 \times 10^{11} \mathrm{~m}^{-3}\right)$ that width is about $20 \mathrm{~mm}$, leading to a cross section area $A_{t}=2 \mathrm{~mm}^{2}$. With $L_{t}=1.35 \mathrm{~km}$, Eq. (19) yields $F(\mathrm{~N})=0.177 \times\left(\gamma+\bar{W}_{e}\right) \eta_{t}$. Choosing $\Lambda_{i}=0.5, \bar{W}_{e}=$ 0.4 in Fig. 2, we get $\eta_{t}=0.3$; this results in a thrust $F=0.032 \mathrm{~N}$. We also find a power $W_{e}=0.54 \mathrm{~kW}$, and a power-system mass $\beta W_{e}=27 \mathrm{~kg}$. Tether mass would be $7.3 \mathrm{~kg}$, the full tether system mass excluding hollow cathode expellant amounting to about $42 \mathrm{~kg}$.

Thrust (but also required power, and tether and power-system masses) can be scaled up, to some degree, by simply scaling up tether width. A 40-mm width (twice maximum value for OML collection, or 8 times Debye length) results in a factor $G=0.97$ in Eq.(9) (Sanmartin and Estes, 2001; Fig.1), non-OML effects being practically negligible; a $60 \mathrm{~mm}$ width would yield $G=0.92$, still very close to 1 . Use of 2 or 3 tapes $20 \mathrm{~mm}$ wide would have no non-OML effects at all, but interference effects (Sanmartin and Estes, 2001) tip the balance in favor of a single tape (40 or $60 \mathrm{~mm}$ wide). A basic point on this issue is that $\lambda_{1}$ is width-independent for a tape, $2 \mathrm{~A}_{1} / \mathrm{p}$ being its

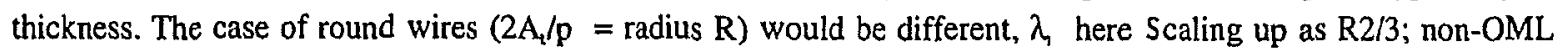
effects might still be negligible, but efficiency is now degraded by size. 


\section{DISCUSSION}

In addition to the propulsive performance of the electrodynamic tether, there are a number of other issues related with the reduction of tether length $L_{t}$ as follows: (a) collision risk posed by the tether to other space vehicles; (b) risk of tether being cut by micrometeoroids and orbital debris; (c) librational dynamic stability of the tether system. In the following, we give a few indications on the advantages of short (order of $1 \mathrm{~km}$ ) tethers vs. long (order of $10 \mathrm{~km}$ ) tethers.

The non-negligible collision risk posed by the tether to other space vehicles (Cooke et al., 2001) depends on the cross section of the tether defined by its length and (if applicable) libration angles. By decreasing the tether length, the risk of impacting other satellites decreases linearly with length for a stationary hanging tether or more than linearly for a librating tether.

The risk of tether being cut depends upon the tether area (and its geometry). The risk of tether failure decreases almost linearly with the tether length in the regime of interest. Moreover, a (hollow) cylindrical tether of 1-kmlength and a few millimeters radius (which is comparable to the Debye length in LEO for nominal conditions) has a survival probability of about $99 \%$ over 2 years lifetime in LEO. A tape of equivalent perimeter may fare somewhat better than the cylindrical configuration.

Lastly, the more complex issue is the dynamic stability of an electrodynamic tether vs. tether length. For this issue we must refer to results by Pelaez et al. (2000), dealing with the simplified case of straight tether with a constant tether current. Under these assumptions, the system exhibits a weak librational instability whose drift rate increases with the ratio of the out-of-plane electrodynamic force over the vertical gravity gradient force. This ratio decreases with tether length for boosting tethers because the gravity gradient force scales with $L_{l}$ whereas the dependance of electrodynamic force on length is more than linear (with a power exponent as high as 2.5 for low resistance tethers); in conclusion the ratio of the two forces decreases as tether length increases. All in all, a short tether is strongly advantageous for the issues (a) and (b) and significantly advantageous for the issue (c).

\section{CONCLUSIONS}

Short electrodynamic boosting tethers (order $1 \mathrm{~km}$ ) have advantages with respect to long (order of $10 \mathrm{~km}$ ) tethers in the areas of collision avoidance and survivabilty to small debris impacts. Although the efficiency is less than that of longer tethers, it is still adequate for several applications when the tether is suitably designed.

\section{ACKNOWLEDGMENTS}

The work of Estes and Lorenzini was supported by NASA Grant NAG8-1605 from the Marshall Space Flight Center. The work of Sanmartin was supported by the Comision Interministerial de Ciencia y Tecnologia of Spain, Grant PB97-0574-C04-1.

\section{REFERENCES}

Cooke, W.J, Spencer, D.B., Anderson, B.J. and Suggs, R.M., "Tether Survivability and Collision Avoidance: Is LEO the Right Place for Tethered Systems?" Procs. of STAIF 2001 (ed. M. El-Genk), AIP, New York (2001).

Estes, R.D., and Sanmartin, J. R., "Cylindrical Langmuir Probes Beyond the Orbital-Motion-Limited Regime," Phys.Plasmas 7, pp. $4320-4325$ (2000).

Martinez-Sanchez, M., and Pollard, J. E., "Spacecraft Electric Propulsion - An Overview," J. Prop. Power 14, pp. $688-699$ (1998).

Pelaez, J., Lorenzini, E. C., Lopez-Rebollal, O., and Ruiz, M., "A New Kind of Dynamic Instability in Electrodynamic Tethers," J. Astron. Sci. 48, pp. 449-476 (2000).

Sanmartin, J. R, Martinez-Sanchez, M., and Ahedo, E., "Bare Wire Anodes for Electrodynamic Tethers," J. Prop. Power 9, pp. 353-360 (1993).

Sanmartin, J. R., and Estes, R. D., "The Orbital-Motion-Limited Regime of Cylindrical Langmuir Probes," Phys.Plasmas 6, pp. 395-405 (1999).

Sanmartin, J. R., Estes, R. D., and Lorenzini, E. C., "Efficiency of Different Types of ED-Tether Thrusters" in Space Technology and Applications International Forum 2001", pp.479-487 (ed. El-Genk) AIP, New York (2001).

Sanmartin, J. R., and Estes, R. D., "Interference of Parallel Cylindrical Langmuir Probes," Phys. Plasmas 8 (September 2001 ). 